\title{
Ketorolac Tromethamine
}

National Cancer Institute

\section{Source}

National Cancer Institute. Ketorolac Tromethamine. NCI Thesaurus. Code C61798.

The tromethamine salt of ketorolac, a synthetic pyrrolizine carboxylic acid derivative with anti-inflammatory, analgesic and antipyretic properties. Ketorolac tromethamine, a nonselective inhibitor of the cyclooxyg enases (COX), inhibits both COX-1 and COX-2 enzymes. This ag ent exerts its anti-inflammatory effect by preventing conversion of arachidonic acid to prostaglandins at inflammation site mediated through inhibition of COX-2, which is undetectable in most tissues but is up-regulated at the inflammation sites. Since COX-1 is expressed virtually in all tissues, inhibition of COX-1 enzyme by this agent prevents normal state production of prostaglandins, which plays housekeeping roles in the protection of the gastrointestinal tract, regulating renal blood flow, and functioning in platelet aggregation. As a result, inhibition of COX-1 is usually associated with adverse effects such as gastrointestinal toxicity and nephrotoxicity. 